\title{
NUTRICIÓN Y CROSSFIT. PLANIFICACIÓN
}

\section{Manuel Navarro Castro}

\section{navarro.castro@alu.uhu.es}

\section{RESUMEN:}

En este trabajo he querido realizar la planificación y la elaboración de la dieta de un deportista de crossfit de nivel intermedio que competirá en el mes de Mayo de forma individual dentro de la categoría masculina. Debido a la ambivalencia de pruebas que encontramos en este deporte, nos centraremos en una ronda de 3 ejercicios básicos y en el formato AMRAP (as many round as posible) durante 10 minutos. El porcentaje de utilización de los alimentos energéticos es muy variable durante la temporada, al igual que el uso de vitaminas y minerales. La temporada comprenderá 4 etapas: Pretemporada, temporada, competición y lesión.

\section{PALABRAS CLAVE:}

Deporte, nutrición, crossfit, planificación, dieta, entrenamiento. 


\section{INTRODUCCIÓN}

El crossfit es un deporte multidisciplinar, aunque en sus inicios se constituyó como un método de entrenamiento, donde se busca el desarrollo corporal trabajando el cuerpo como un conjunto mediante pruebas físicas de otros deportes. Hoy en día cada vez más se aprecia el peso de la nutrición en los deportistas, debido a esto y respecto a la escasa existencia de trabajos y análisis en habla hispana sobre esta temática, recurriremos a estudios en habla anglosajona y referencias genéricas existentes, y a su vez analizaremos los aspectos más relevantes que tengan relación con estos 3 ejercicios ya que son los más representativos (M.Smith, J.Sommer, E.Starkoff, \& T.Devor, n.d.) y en base a ellos elaboraremos una dieta para cumplir unos objetivos en durante una sola temporada:

- Squat.

- Power snatch.

- Kipping pull up.

En este deporte tanto las pruebas como la duración de las mismas son muy variables por lo que se realizará un uso de los dos sistemas de energía, la anaeróbica láctica y aeróbica. Hay que puntualizar la última vía mencionada, la aeróbica, hay pocas pruebas en las que se use dentro del crossfit y además en este trabajo no se va a tratar, ya que hemos elegido formato “As Many Round As Possible” (AMRAP) durante 10 minutos realizando 10 "squats", 10 “power snatch" y 10 "kipping pull up", por lo que los objetivos en la nutrición expuesta es principalmente para apoyar la primeras vía, además que debido a las pruebas elegidas no será un aspecto potencial a estudiar de manera profunda. 


\section{METODOLOGÍA}

En este apartado hablaremos en primer lugar sobre las características del sujeto, que mediante una impedancia bioeléctrica hemos podido señalar los aspectos claves a mejorar (agosto de 2014):

- Peso: 82'1 kg.

- Altura: $180 \mathrm{~cm}$.

- Masa grasa: 11’4 kg.

- Porcentaje de grasa corporal: 13 '9\%.

- Porcentaje de agua corporal: 39'9\%.

- Masa libre de grasa: 70'7 kg.

- Porcentaje masa libre de grasa: 86'1\%.

- Masa muscular: 40’8 kg.

- Porcentaje masa muscular: 49'69\%.

- Agua intracelular: 32'8 kg.

- Agua extracelular: 18'9 kg.

- Masa ósea: 4’8 kg.

- Perímetro brazo: $32 \mathrm{~cm}$.

- Perímetro cintura: 84’5 cm.

- Perímetro cadera: 98’5 cm.

Para calcular el gasto calórico total, incluyendo la vida diaria del deportista y su entrenamiento, utilizaremos la ecuación elaborada por la Organización Mundial de la Salud (OMS, 1985) sobre el gasto energético en reposo (GER - exactamente lo mismo que el metabolismo basal) por un factor basado en el nivel de actividad y por otro de clima: 
- Cantidad kcal totales: $1846 \mathrm{kcal}$ (Metabolismo basal) * "x" (nivel de actividad intenso) *1'1 (factor termogénico alimentos 10\%) * “y” (factor clima)

- Las componentes en color azul indican una variabilidad de la energía necesaria diaria según el periodo en el que nos encontremos y la actividad que se realice.

○ $\mathrm{Y}=1$ '1 si las temperaturas determinan sustancialmente la cantidad de energía.

En base a estos datos, podremos establecer las necesidades totales dietéticas que precise el deportista para una ronda tipo AMRAP durante 10 minutos.

En segundo lugar estableceremos los objetivos y su planificación temporal para cada estadio, partiendo del punto de vista del entrenamiento hacia el punto de vista nutricional:

1. Recuperación: Periodo precompetitivo en el que iremos adaptando la condición física actual del sujeto a los factores limitantes de las pruebas, lo que comprenderá momentos en los que aumentaremos el número de kcal a ingerir para asegurar el anabolismo necesario.

- Asegurar una correcta técnica de cada ejercicio.

- Controlar la subida del \% graso del sujeto.

- Habituar al deportista a usar determinados patrones nutricionales.

- Desarrollar paralelamente la fuerza explosiva y la resistencia velocidad máxima.

○ Hipertrofia muscular, principalmente sarcomérica, como una de las vías para ganar fuerza.

- Aumentar la masa muscular hasta acercarnos a las necesidades físicas de cada prueba (tendencia a mesomorfismo). 
2. Inicio de temporada: Periodo que comprende desde que la recuperación ha culminado, hasta el primer día de competición, por lo que se debe trabajar en paralelo la nutrición y la programación del entrenamiento, es decir, trabajar a la vez el entrenador y el nutricionista.

a. Mantener y aumentar hacia un nivel óptimo las capacidades físicas $\underline{\text { mantener equidad respecto a las fuentes energética para mantener el }}$ rendimiento:

i. Fuerza explosiva.

ii. Resistencia muscular.

b. Disminuir poco a poco el porcentaje de la grasa corporal para mejorar la eficiencia del sujeto - dieta con tendencia a hipocalórica.

3. Competición: Periodo en el que las cualidades físicas deben estar o acercarse (si existen varias competiciones cercanas) a su máximo rendimiento.

a. Mantener los niveles de masa muscular esquelética y masa grasa constantes - dieta equilibrada calóricamente.

b. Facilitar la recuperación tanto en la competición como al terminar recuperación electrolitos y niveles de fluido. 
4. Lesión: Periodo en el que el deportista ha sufrido un golpe el cual le impide expresar su máximo rendimiento.

a. Grupos musculares que pueden verse afectados o sobreestresados:

1. En el ejercicio "kipping pull up" carecemos de bibliografía específica, pero estudios como Koukoubis et al (1995) sobre los músculos del brazo implicados en la escalada dice que la mayoría de las lesiones se producen en el supinador largo y en el flexor superficial.

2. En el ejercicio "squat" (sentadilla en castellano), Lavorato y Pereira (2009) explican que las lesiones durante la sentadilla vienen producidas por una mala ejecución técnica (incluso si son sentadilla profundas) por lo que teniendo un deportista de nivel intermedio de crossfit, con un periodo de la temporada enfocado específicamente en la correcta ejecución técnica, sobreentendemos que los músculos que están implicados aquí no precisan una especial atención.

3. En el ejercicio "power snatch" Konig y Biener (1990) resaltan que la mayoría de las lesiones en levantadores olímpicos provienen de lesiones en la rodilla (25\%), cintura escapular (22\%), espalda (21\%), muñeca (12\%) y en los codos.

ii. Debido a estos estudios, intentaremos optimizar la nutrición para la recuperación de los músculos que se encuentran en el antebrazo.

b. Aplicar pautas nutricionales que mejoren la recuperación.

i. Vitaminas potenciadoras para la recuperación (Vit. C, grupo B y vit. E).

ii. Minerales necesarios.

c. Controlar en la medida de lo posible la pérdida de masa muscular y la perdida/ganancia de masa grasa. 


\section{RESULTADOS}

Según la ecuación de Harris-Benedict, revisada por Mifflin y St Jeor en 1990, el sujeto presenta una tasa metabólica basal de $1846 \mathrm{kcal} /$ día.

Previamente a la elaboración de la dieta, suministraremos al deportista un cuestionario de su dieta normal durante un periodo de 3 días, en busca de conseguir recoger información sobre los hábitos alimenticios del deportista, he aquí el resultado:

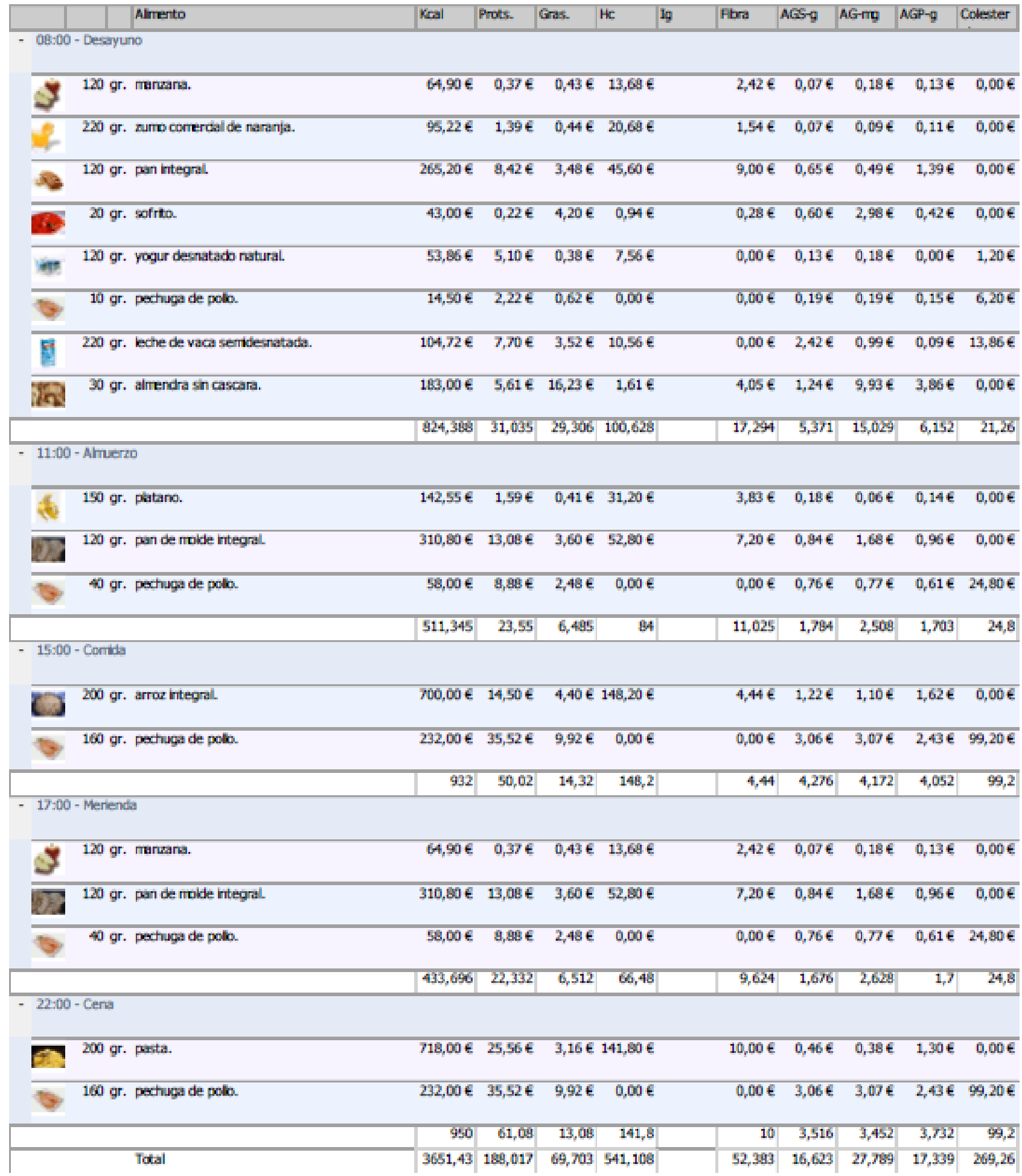




\section{Conclusiones}

Hay que destacar que el sujeto sigue un régimen estricto de comidas y debido a esto se apreció que la composición nutricional de los 3 días era exactamente la misma, razón por la cual solo se presenta la ingesta durante un día. Hay varios aspectos que llaman especialmente la atención:

- No hay un control sobre la cantidad de agua que bebe al día, lo que puede conllevar a una mala o incorrecta distribución del líquido durante la jornada.

- Al mismo tiempo, no hay ningún tipo de hidratación con sales minerales durante el ejercicio, lo que puede conllevar no mantener el máximo rendimiento durante las sesiones.

- Se aprecia que la relación macronutrientes - kcal necesarios está bien enfocada, ya que no existe ningún exceso como se puede apreciar en el gráfico y se asemeja a las recomendaciones que pueden verse a continuación, pero respecto a las vitaminas puede tener ciertos problemas según la época del año o si se encuentra en una lesión, por lo que ser tan estricto con los tipos de alimentos a ingerir no parece ser la mejor elección.

- El sujeto ha consumido $3651 \mathrm{kcal}$, repartidas en:

○ 188 gr de proteínas.

- Ligeramente por encima de las recomendaciones máximas de la OMS (2004) de $2 \mathrm{~g} / \mathrm{kg}$ peso.

○ 70 gr en grasas.

○ 541 gr en hidratos de carbono.

\section{KCAL TOTALES - REPARTO MACRONUTRIENTES}

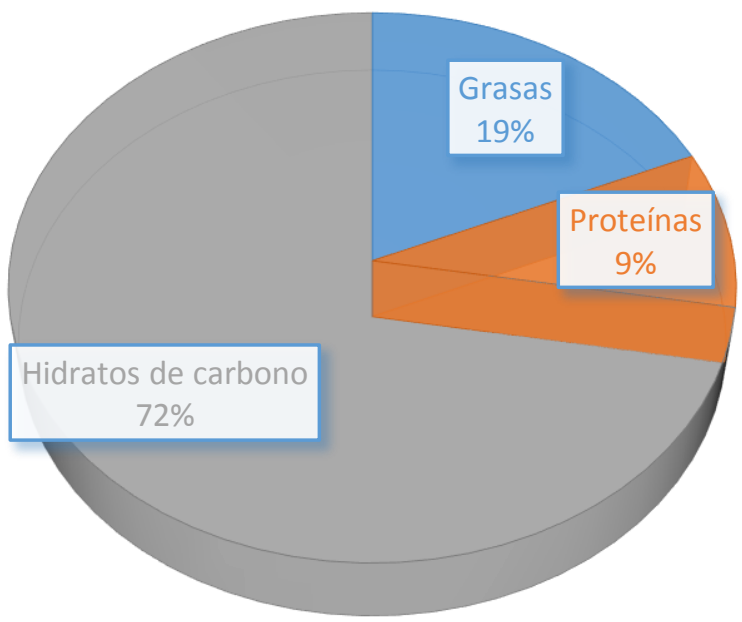




\section{- Pautas generales de actuación en la intervención de la dieta:}

- Debido a que no queremos establecer un gasto económico elevado, se estrecha el uso de suplementación dietética.

- Recomendación general de kcal en función del deporte:

- No utilizaremos ninguna recomendación general para deportes de alta intensidad ya que la mayoría de ellos, como por ejemplo la halterofilia, tiene unos periodos de actividad menores y periodos de descansos mayores, y a falta de estudios concretos en crossfit, preferimos utilizar las ecuaciones expuestas anteriormente para cada periodo.

- El sujeto presentaba un total descontrol sobre las ingestas de agua, por lo que debemos educar al deportista para que conozcas algunas pautas:

- La American College of Sports Medicine (1996), aconseja consumir $500 \mathrm{ml}$ de fluido durante las 2 horas previas al ejercicio.

- Castillo, Gutiérrez, Mesa, Mula, y Ruiz (2003) recomiendan en los momentos previos a la competición tomar bebidas ricas en carbohidratos para aumentar la disponibilidad periférica de la glucosa y frenar su producción hepática, lo que al final conlleva una aparición más tardía de la fatiga. Al mismo tiempo establecieron que con un trabajo corporal intenso en un clima moderado, se aconseja una ingesta de líquido de 300 - $500 \mathrm{ml}$ con un $6-10 \%$ de concentración de carbohidratos, cada 15 minutos y a una temperatura de $5-15^{\circ}$.

- Durante la temporada y antes de competir hay que realizar una disminución del peso corporal para tener el máximo porcentaje de músculo para el peso en el que compitamos, mediante una dieta baja en carbohidratos. Hay que destacar que al no haber categoría por peso en estas pruebas, no hay que realizar una disminución de la ingesta de fluido.

- Los porcentajes de utilización de cada sustrato energético según Baechle y Earle (2008) para actividades intensas son:

○ $60-70 \%$ hidratos de carbono.

- $\mathrm{Al}$ menos un $15-20 \%$ de grasas.

○ $10-15 \%$ proteínas.

- En cantidades exactas, se recomienda para deportes resistidos una cantidad 1,5 - $2 \mathrm{gr} * \mathrm{~kg}$ corporal. 
- Periodo de ganancia de peso:

- La NSCA (2008) recomienda un superávit de entre 350 y 700 kcal por encima de los requisitos diarios para mantener entre 450 y 900 gramos semanales de tejido magro.

○ Consejos:

- Comer más veces (aunque no es del todo recomendable debido a las digestiones).

- Consumir mayores porciones de alimentos.

- Elegir alimentos más ricos en kilocalorías.

- Periodo de pérdida de peso:

- La NSCA (2008) recomienda que las pérdidas sean del $1 \%$ del peso corporal a la semana para que no existan problemas de deshidratación ni reducción del nivel de vitaminas y minerales.

- La NSCA (2008) recomienda un déficit de entre 500 - 1000 kcal semanales.

○ Consejos:

- Consumir alimentos de poca densidad energética.

- Dado que en la termogénesis de los alimentos se pierde, según Nieto Martínez (s.f), alrededor de un $10 \%$ hay que contar con sumar un $10 \%$ del total de kcal necesarias para cada periodo, ya que las ecuaciones utilizadas en este trabajo no tienen en cuenta este factor.

- Somatotipo, porcentajes de grasa:

- Según España-Romero et al (2009) en un estudio sobre las características de los escaladores, los porcentajes de grasa se sitúan en torno al 9-10\% del peso corporal total, siendo menor cuanto más tiempo se situé el cuerpo en el aire en contra de la gravedad.

- Dado que el somatotipo corporal que presentan comúnmente los deportistas de crossfit se aproxima al de los halterófilos, según Martínez de Haro (2010) la mesomorfia y la endomorfia son dos factores claves a controlar, mientras que la ectomorfia es menos determinante.

- Al final de este punto adjuntamos la somatocarta actual del deportista. 


\section{Somatocarta}

Según las ecuaciones de Cabañas y Armesilla (2009) citado por Martínez-Sanz et al, podemos calcular el grado de endomorfismo, mesomorfismo y ectomorfismo:

\begin{tabular}{|c|c|c|}
\hline Componente & Formula & Información \\
\hline Ectomorfia & $\begin{array}{l}-0,7182+0,1451^{*} \mathrm{X}-0,00068^{*} \mathrm{X}^{2}+ \\
0,0000014^{*} \mathrm{X}^{3}\end{array}$ & $\begin{array}{l}\mathrm{X}=(\mathrm{Pl} \text { Triceps }+\mathrm{PI} \text { Subescapular }+ \\
\mathrm{PI} \text { Suprailiaco })^{*}(170,18 / \text { Estatura }) \\
\text { Estatura en } \mathrm{cm}\end{array}$ \\
\hline Mesomorfia & $\begin{array}{l}\left(0,858^{*} \mathrm{DH}+0,601^{*} \mathrm{DF}+\right. \\
\left.0,188^{*} \mathrm{PBC}+0,161^{*} \mathrm{PGC}\right)- \\
(\text { estatura*0 } 0,131)+4,5\end{array}$ & $\begin{array}{l}\text { DH= diámetro del humero en } \mathrm{cm} \\
\mathrm{DF}=\text { diámetro del fémur en } \mathrm{cm} \\
\mathrm{PBC}=\text { perimetro del brazo relajado } \\
\text { corregido } \\
\text { PGC=perimetro de gemelar o de la } \\
\text { pantorrilla corregido } \\
\text { Estatura en } \mathrm{cm}\end{array}$ \\
\hline Ectomorfia & $\begin{array}{l}\text { SI IP } \geq 40,75 \rightarrow=\left(0,732^{*} \mathrm{IP}\right)-28,58 \\
\text { Si IP entre } 38,25-40,75 \rightarrow= \\
\left(0,463^{*} \mathrm{IP}\right)-17,63 \\
\text { Si IP } \leq 38,25 \rightarrow=0,1\end{array}$ & $\begin{array}{l}\text { Se requiere el cálculo del CAP o } \\
\text { indice ponderal (IP) para utilizar } \\
\text { una fómula u otra: } \\
\text { CAP o IP= estatura }(\mathrm{cm}) / 3 \text { vpeso } \\
\text { (raiz cúbica del peso en } \mathrm{kg} \text { ) }\end{array}$ \\
\hline
\end{tabular}

Tabla 1. Apuntes no publicados del profesor Don Eduardo Fernández Ozcorta.

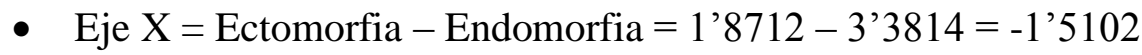

- Eje $\mathrm{Y}=2 *$ Mesomorfia - Endomorfia - Ectomorfia $=2 * 3{ }^{\prime} 11-3 ’ 3814-1.8712$

$=0 ’ 9674$

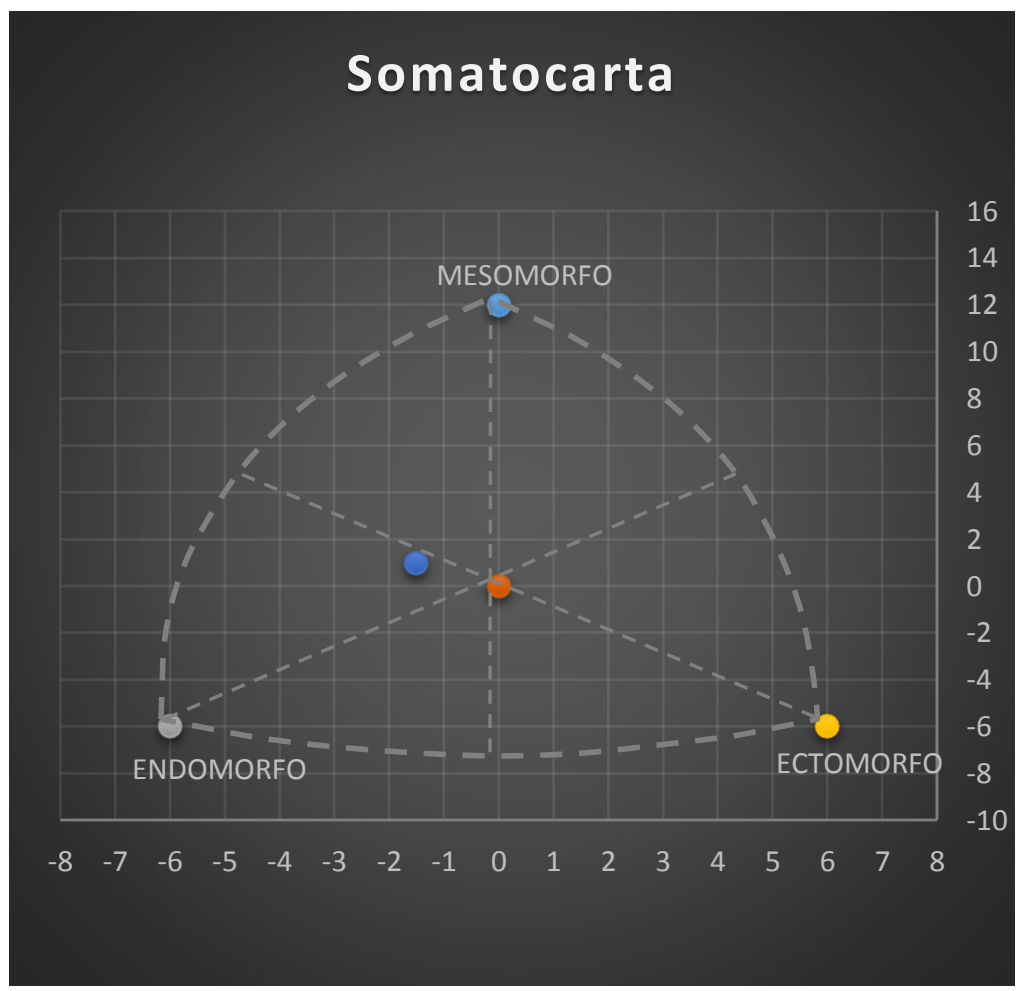


Dieta periodo 1: Recuperación (Septiembre - Octubre).

Durante este periodo como he mencionado anteriormente, debemos tener un superávit calórico para favorecer el anabolismo. Igualmente, el porcentaje de ingesta de la grasa debe ser alto para favorecer altos niveles de testosterona (M.Boetes, J.A.Bush, T.Incledon, W.J.Kraemer, J.S.Volek, 1997). Las condiciones ambientales no son factores modificantes por ahora. Los 4 días elegidos serán:

- Lunes: Entrenamiento de musculación.

- Martes: Entrenamiento de musculación.

- Miércoles: Descanso.

- Jueves: Entrenamiento de musculación.

Necesidades calóricas días de entreno: 1846 kcal/día *2,1 (intensidad - factor térmico ejercicio $) * 1,1($ factor termogénico $10 \%)=4264.26 \mathrm{kcal}+350 \mathrm{kcal}($ superávit $)=$ $4614.26 \mathrm{kcal}$

Necesidades calóricas días de descanso: 1846 kcal/día * 1,6 * 1,1 = 3248.96 kcal + 350

$$
\mathrm{kcal}=3598.96 \mathrm{kcal}
$$

Reparto de macronutrientes:

$$
\begin{aligned}
& \text { 65\% Hc; 20\% Grasas; 15\% Proteína } \\
& 749 \text { g Hc; } 102 \text { g Grasas; } 173 \text { g Proteína } \\
& 584 \text { g Hc; } 80 \text { g Grasas; } 134 \text { g Proteína }
\end{aligned}
$$




\section{Lunes}

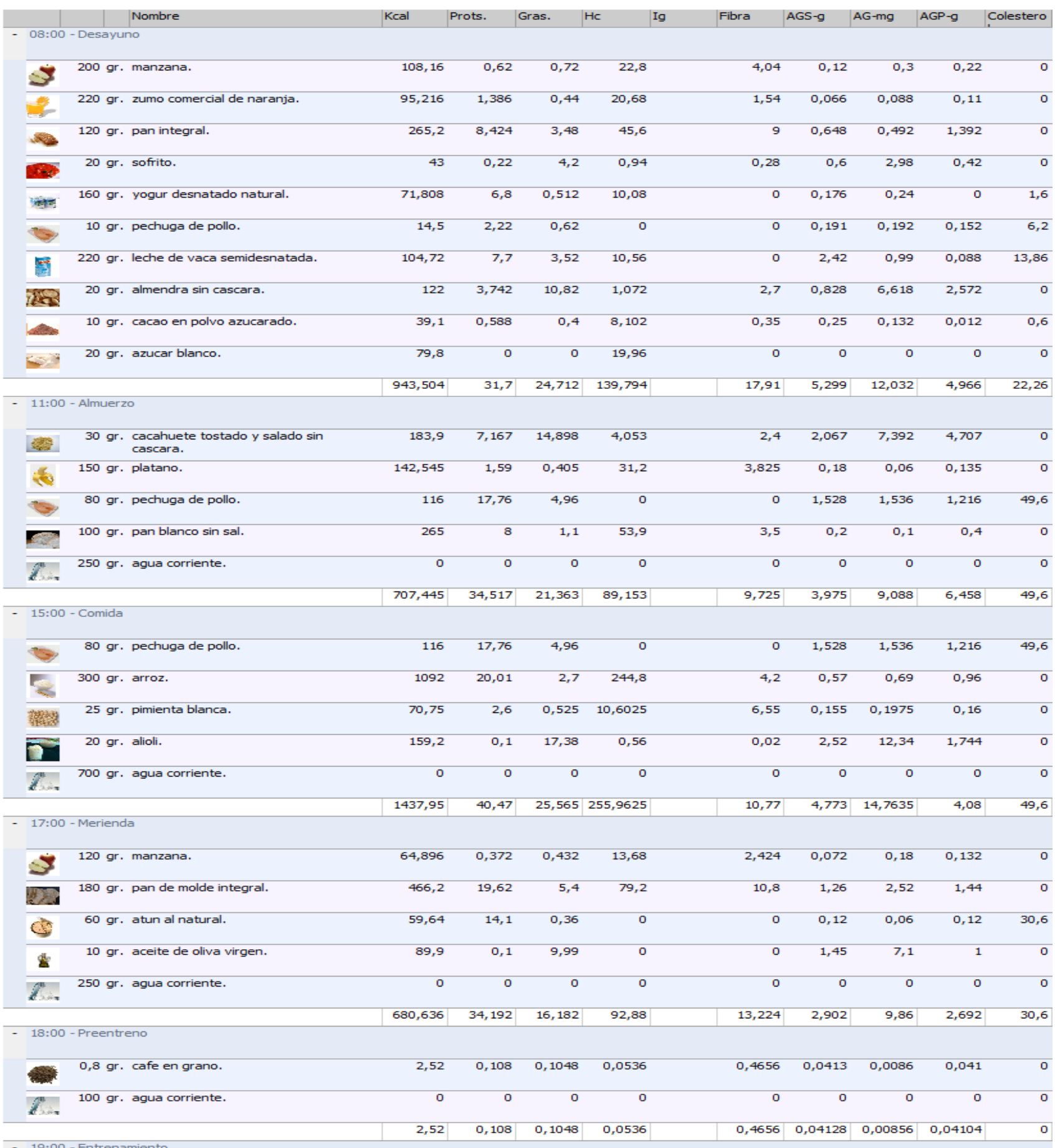

\section{IfI} 200 Powerade ION4 34 7,8

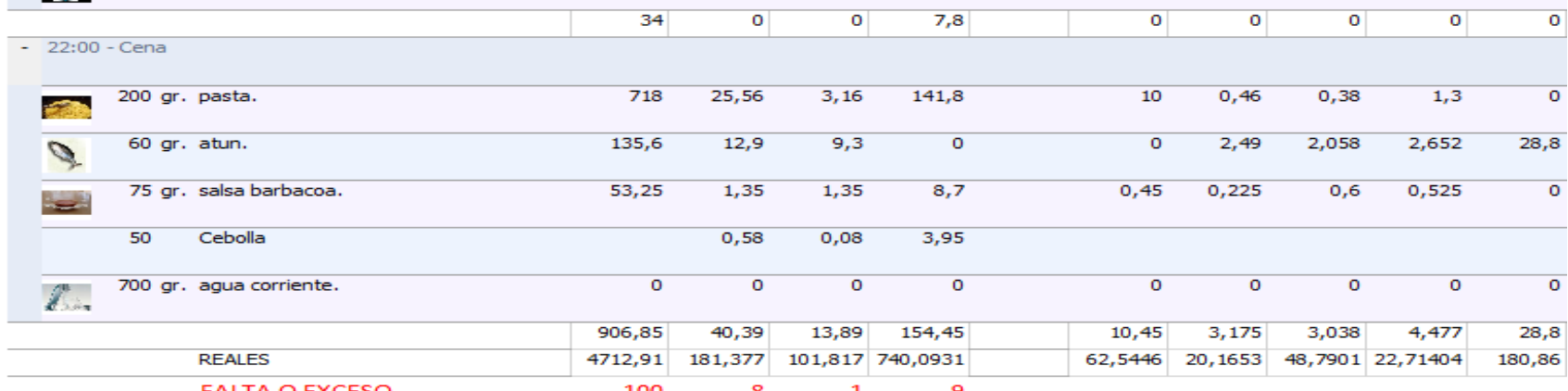

FALTA O EXCESO

Número 3, 2014
E-motion. Revista de Educación, Motricidad e Investigación 


\section{Martes}

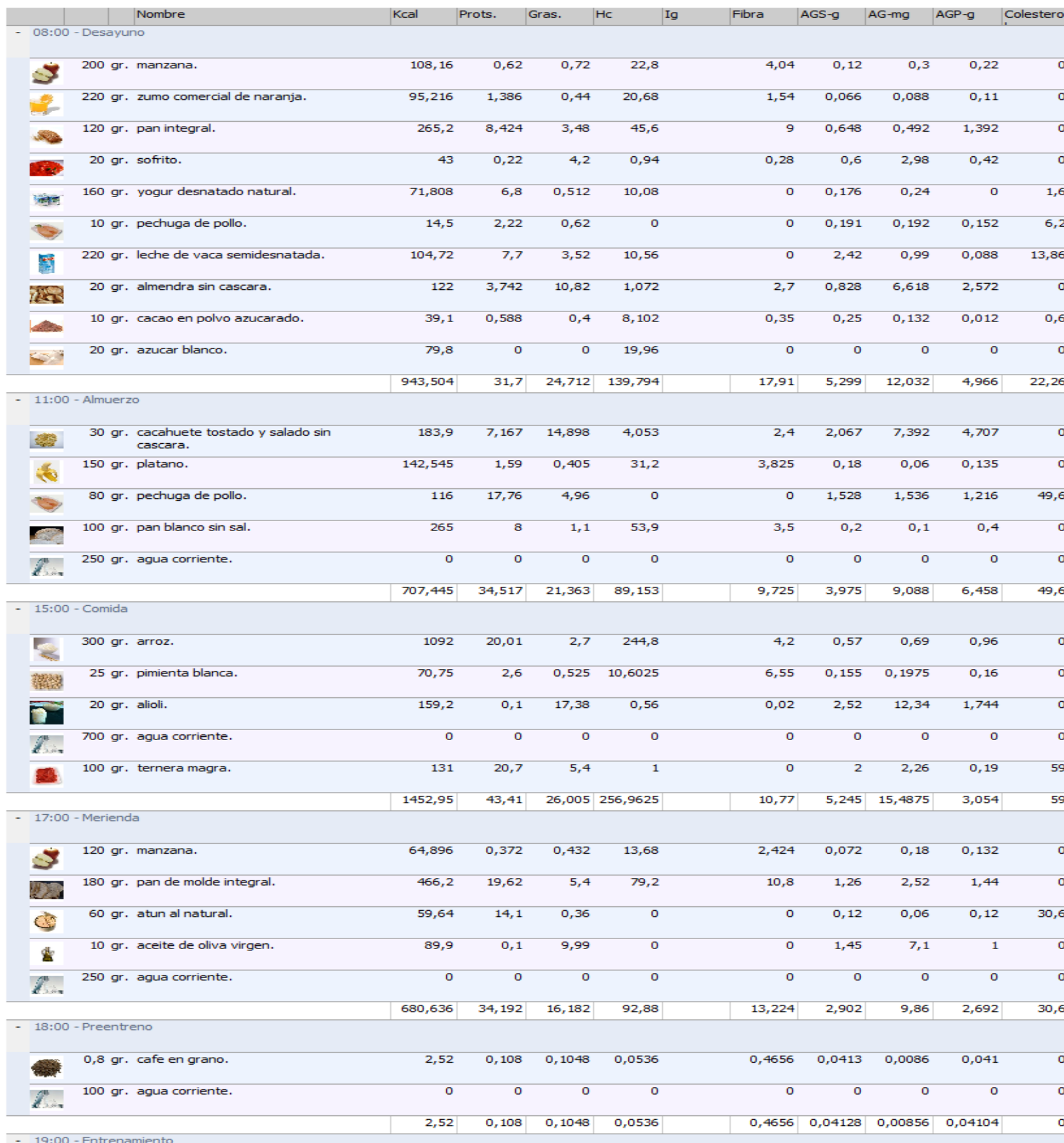

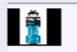

\section{$34+7,8$}

\begin{tabular}{|c|c|c|c|c|c|c|c|c|c|c|c|}
\hline \multicolumn{12}{|c|}{ - 22:00 - Cena } \\
\hline$\infty$ & $200 \mathrm{gr}$. & pasta. & 718 & 25,56 & 3,16 & 141,8 & 10 & 0,46 & 0,38 & 1,3 & 0 \\
\hline & 50 & Cebolla & & 0,58 & 0,08 & 3,95 & & & & & \\
\hline b. & $700 \mathrm{gr}$. & agua corriente. & 0 & 0 & 0 & 0 & 0 & o & 0 & 0 & 0 \\
\hline $55^{3}$ & $100 \mathrm{gr}$. & pescaditos. & 79,8 & 11,4 & 3 & 1,8 & 0 & 1,15 & 0,4 & 1,15 & 34,5 \\
\hline 0 & $50 \mathrm{gr}$. & salsa de queso. & 55,5 & 2,65 & 3,05 & 4,2 & 0,25 & 1,285 & 0,85 & 0,6 & 14,5 \\
\hline & & & 853,3 & 40,19 & 9,29 & 151,75 & 10,25 & 2,895 & 1,63 & 3,05 & 49 \\
\hline & & REALES & 4674,36 & 184,117 & 97,6568 & 738,3931 & 62,3446 & 20,3573 & 48,1061 & 20,26104 & 210,46 \\
\hline
\end{tabular}


Miércoles

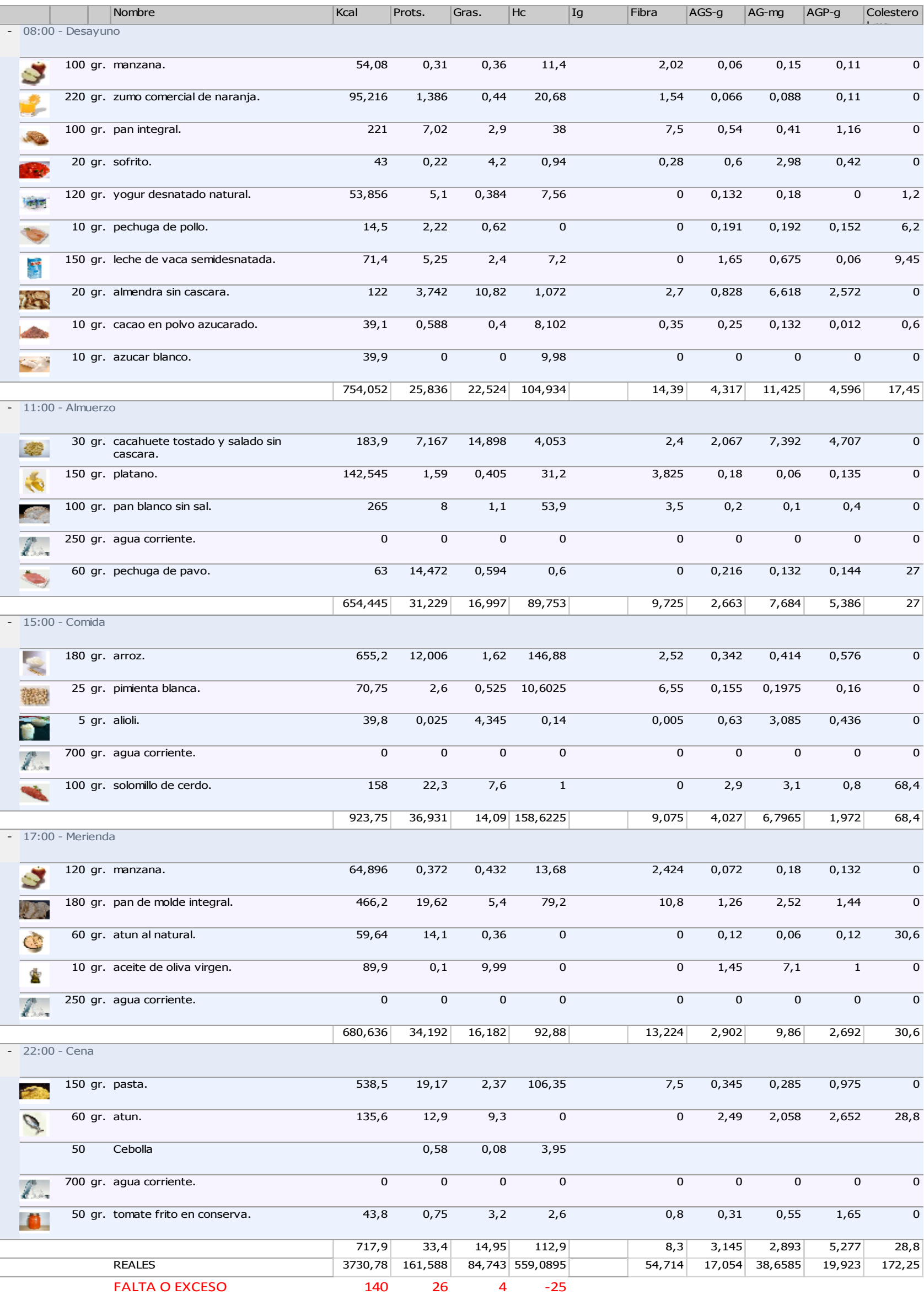




\section{Jueves}

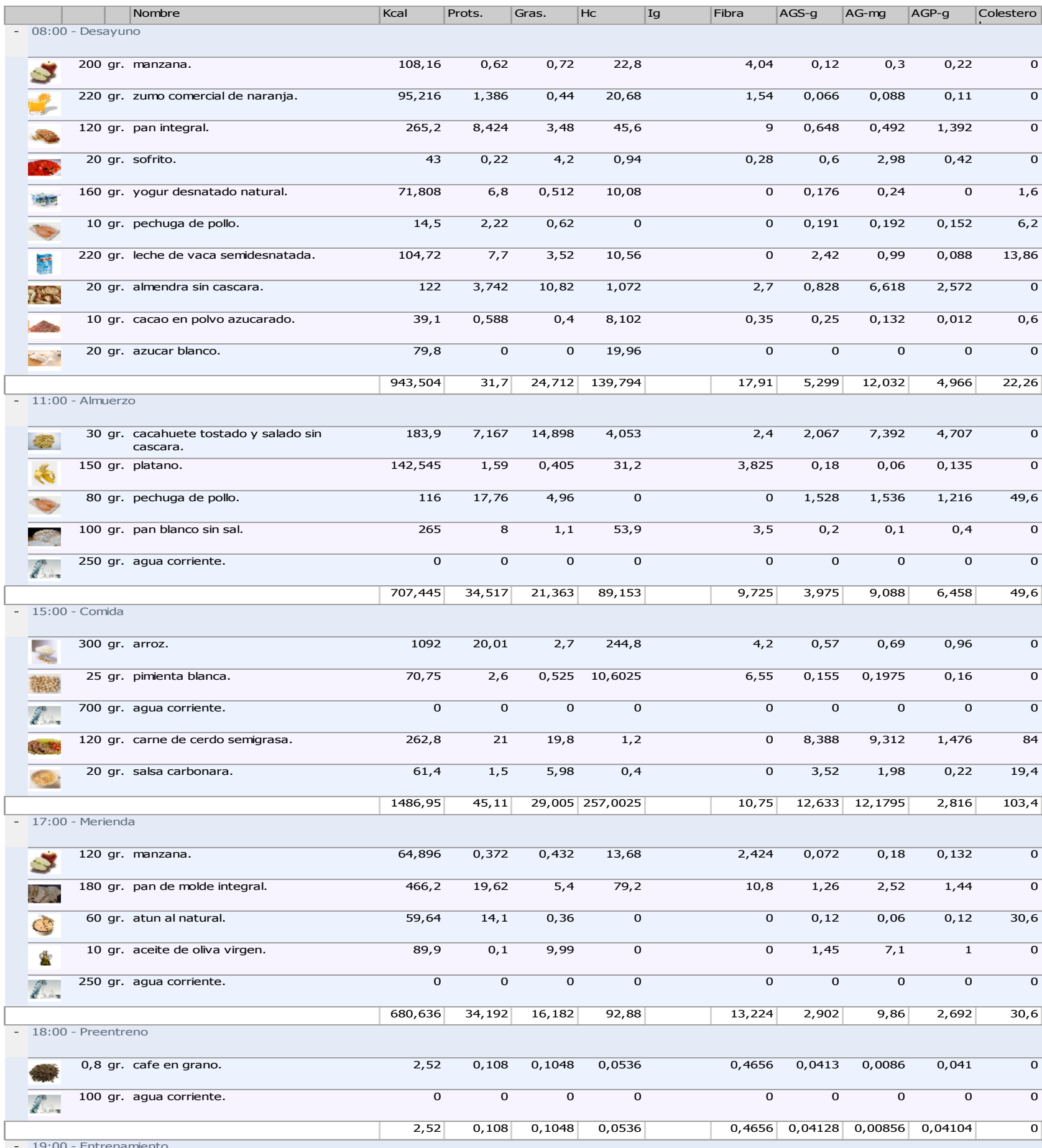

\section{III} $\begin{array}{llll}200 & \text { Powerade ION4 } & 34 & 7,8\end{array}$

\begin{tabular}{|c|c|c|c|c|c|c|c|c|c|c|}
\hline & & 34 & 0 & 0 & 7,8 & 0 & 0 & 0 & 0 & 0 \\
\hline \multicolumn{11}{|c|}{ - 22:00 - Cena } \\
\hline \multirow{2}{*}{$=$} & 75 gr. salsa barbacoa. & 53,25 & 1,35 & 1,35 & 8,7 & 0,45 & 0,225 & 0,6 & 0,525 & 0 \\
\hline & Cebolla & & 0,58 & 0,08 & 3,95 & & & & & \\
\hline $6=$ & 700 gr. agua corriente. & 0 & 0 & 0 & 0 & 0 & 0 & 0 & 0 & 0 \\
\hline 3 & 200 gr. pasta rellena de carne hervida. & 208 & 9,4 & 6,8 & 25,4 & 3,6 & 2,6 & 3,2 & 0,6 & 46 \\
\hline 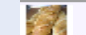 & 200 gr. pan blanco tipo baguette. & 516 & 19,3 & 2,2 & 101,2 & 7 & 0,4 & 0,2 & 0,8 & 0 \\
\hline & & 777,25 & 30,63 & 10,43 & 139,25 & 11,05 & 3,225 & 4 & 1,925 & 46 \\
\hline & REALES & 4632,31 & 176,257 & 101,797 & 725,9331 & 63,1246 & 28,0753 & 47,1681 & 18,89804 & 251,86 \\
\hline
\end{tabular}


- Dieta periodo 2: Inicio de la temporada (Octubre - Abril).

Durante este periodo como he mencionado anteriormente, debemos tener un leve déficit calórico para ir disminuyendo el porcentaje graso. Los hidratos de carbono vuelven a recuperar importancia para mantener, o mejorar el rendimiento. En algunos meses por condiciones climáticas habrá que sumarle un $10 \%$ del metabolismo basal en concepto del clima (OMS, 1985), el cual incrementa el consumo. Las ecuaciones utilizadas abajo serían para los meses de octubre, febrero, marzo y abril en los que no es necesaria ese aumento. Los 4 días elegidos serán:

- Lunes: Entrenamiento de musculación.

- Martes: Entrenamiento de musculación (High Interval Power Training).

- Miércoles: Descanso.

- Jueves: Entrenamiento de musculación.

Necesidades calóricas días de entreno: 1846 kcal/día * 2,1 (intensidad - factor térmico ejercicio $) * 1,1($ factor termogénico $10 \%)=4264.26 \mathrm{kcal}-500 \mathrm{kcal}($ déficit $)=3764.26$ kcal

Necesidades calóricas días de descanso: 1846 kcal/día * 1,6 * 1,1 = 3248.96 kcal $500 \mathrm{kcal}=2748.96 \mathrm{kcal}$

Reparto de macronutrientes:

70\% Hc; $15 \%$ Grasas; $15 \%$ Proteína

658 g Hc; 63 g Grasas; 141 g Proteína

481 g Hc; 46 g Grasas; 103 g Proteína 


\section{Lunes}

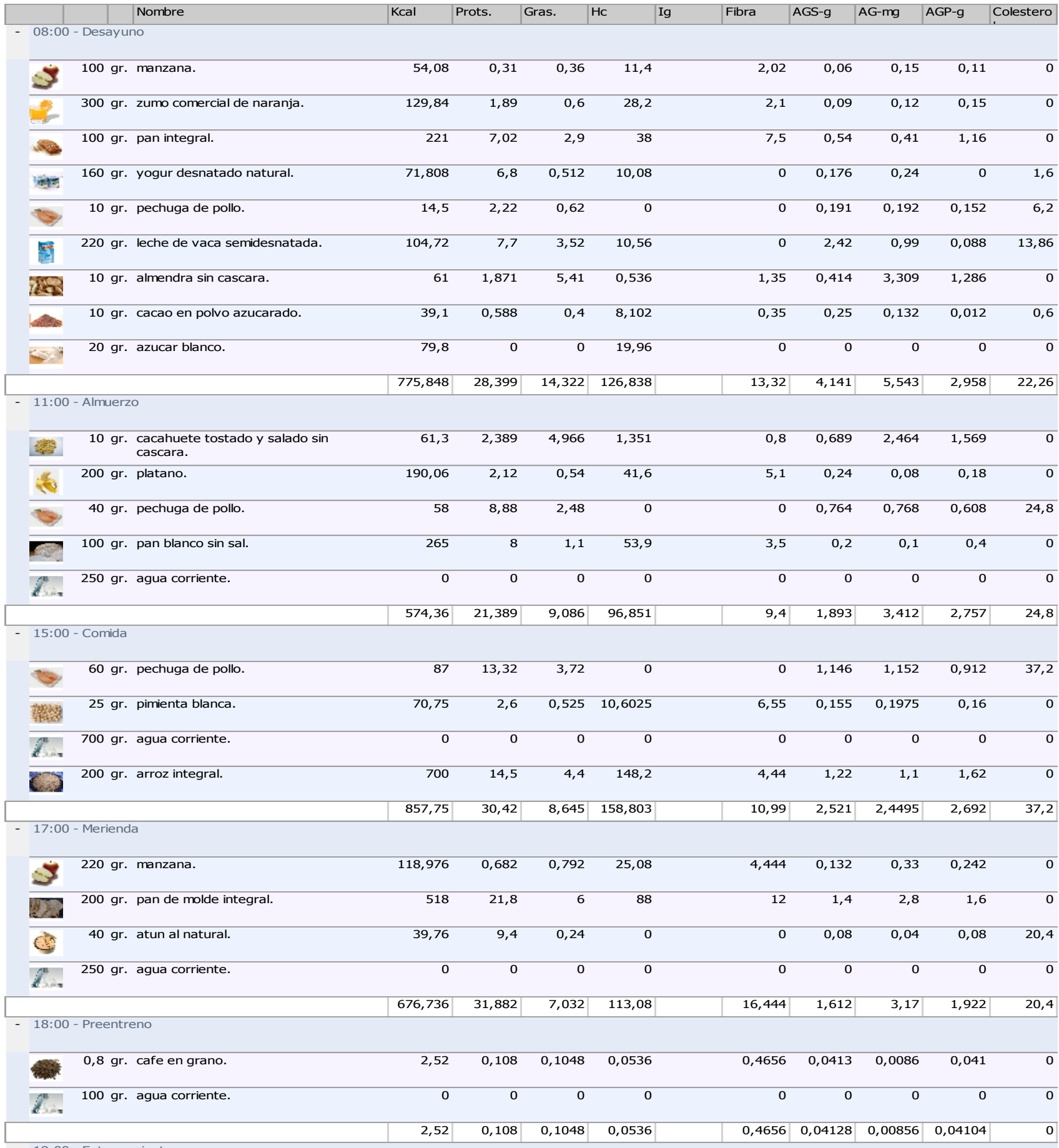

19:00 - Entrenamiento

解 200 Powerade ION4

$34 \quad 7,8$

- 22:00 - Cena

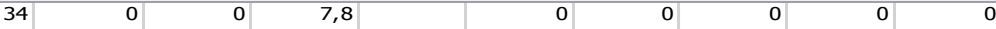

$\begin{array}{llll}49,7 & 1,26 & 1,26 & 8,12\end{array}$

$\begin{array}{lllll}0,42 & 0,21 & 0,56 & 0,49 & 0\end{array}$

50 Cebolla

$0,58 \quad 0,08 \quad 3,95$

है: 700 gr. agua corriente.

$\begin{array}{llllllll}0 & 0 & 0 & 0 & 0 & 0 & 0 & 0\end{array}$

210 gr. pasta al huevo.

$\begin{array}{llll}785,4 & 25,935 & 8,82 & 146,79\end{array}$

$\begin{array}{lllll}7,098 & 1,89 & 3,15 & 0,84 & 63\end{array}$

REALES

FALTA O EXCESO

Número 3, 2014

\begin{tabular}{|r|r|r|r|}
\hline 835,1 & 27,775 & 10,16 & 158,86 \\
\hline
\end{tabular}

\begin{tabular}{|r|r|r|r|r|}
\hline 7,518 & 2,1 & 3,71 & 1,33 & 63 \\
\hline
\end{tabular}

\begin{tabular}{|r|r|r|r|r}
3756,31 & 139,973 & 49,3498 & 662,285
\end{tabular} \begin{tabular}{|l|l|l|}
$\mathbf{5} 8,1376$ & 12,3083 & 18,293 \\
\hline
\end{tabular}

\begin{tabular}{l|l|l|l}
11,7 & 167,66
\end{tabular}

E-motion. Revista de Educación, Motricidad e 
Martes

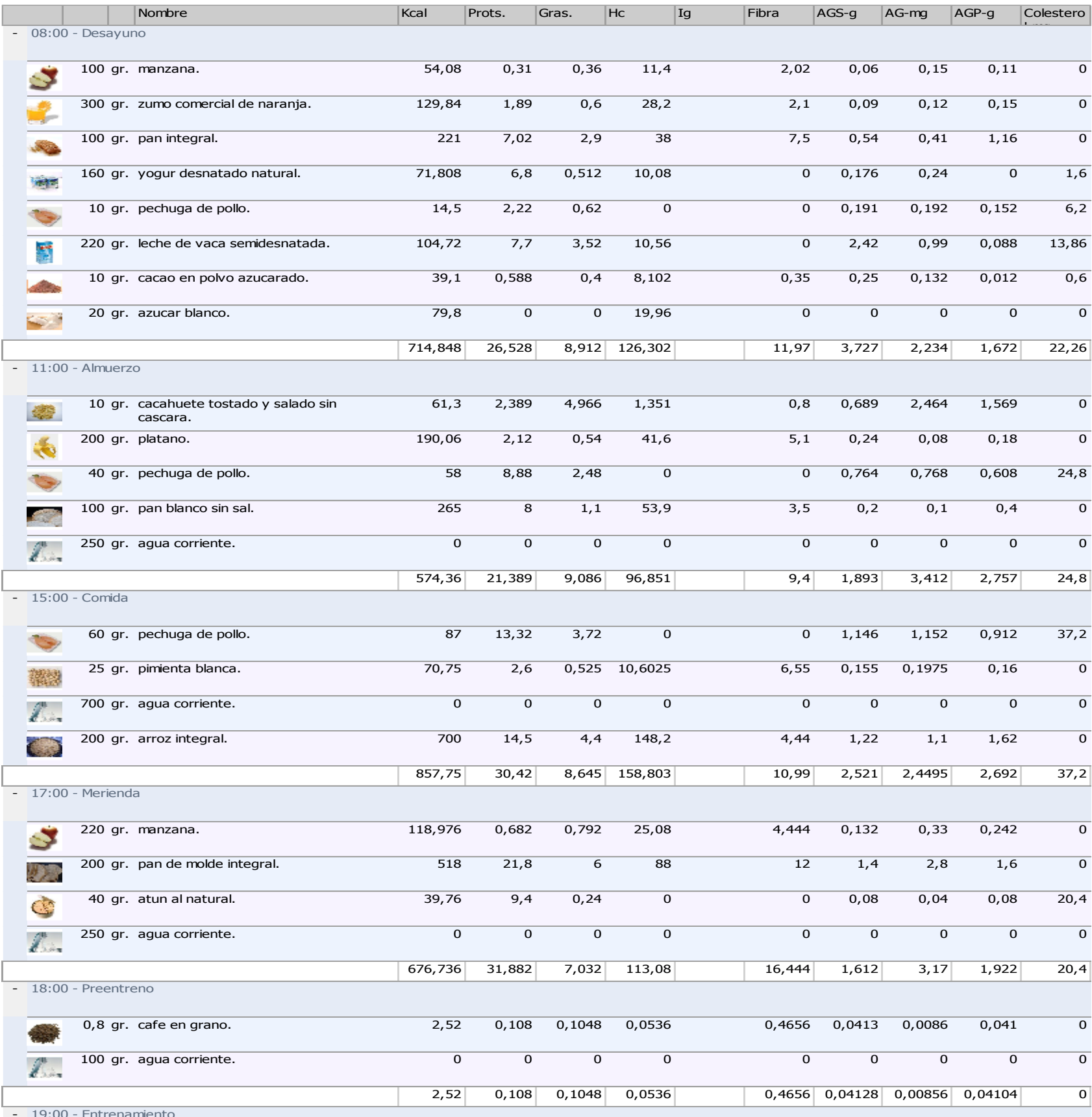

雼 200 Powerade ION4

$34 \quad 7,8$

\begin{tabular}{|c|c|c|c|c|c|c|c|c|c|c|}
\hline & & 34 & 0 & 0 & 7,8 & 0 & 0 & 0 & 0 & 0 \\
\hline \multicolumn{11}{|c|}{ - 22:00 - Cena } \\
\hline \multirow{2}{*}{$=$} & 70 gr. salsa barbacoa. & 49,7 & 1,26 & 1,26 & 8,12 & 0,42 & 0,21 & 0,56 & 0,49 & 0 \\
\hline & 50 Cebolla & & 0,58 & 0,08 & 3,95 & & & & & \\
\hline 6in & 700 gr. agua corriente. & 0 & 0 & 0 & 0 & 0 & 0 & 0 & 0 & 0 \\
\hline REA & 250 gr. patata nueva. & 183,975 & 5,85 & 0,275 & 37 & 5,175 & 0,075 & 0 & 0,15 & 0 \\
\hline 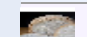 & 175 gr. pan blanco sin sal. & 463,75 & 14 & 1,925 & 94,325 & 6,125 & 0,35 & 0,175 & 0,7 & 0 \\
\hline Qn & 200 gr. naranja. & 90,96 & 1,74 & 0,4 & 17,8 & 4,6 & 0,06 & 0,1 & 0,16 & 0 \\
\hline 5 & 50 gr. muslo de pollo. & 91,5 & 10,31 & 5,6 & 0 & 0 & 1,835 & 1,61 & 1,3 & 43 \\
\hline & & 879,885 & 33,74 & 9,54 & 161,195 & 16,32 & 2,53 & 2,445 & 2,8 & 43 \\
\hline & REALES & 3740,1 & 144,067 & 43,3198 & 664,084 & 65,5896 & 12,3243 & 13,7191 & 11,884 & 147,66 \\
\hline
\end{tabular}




\section{Miércoles}

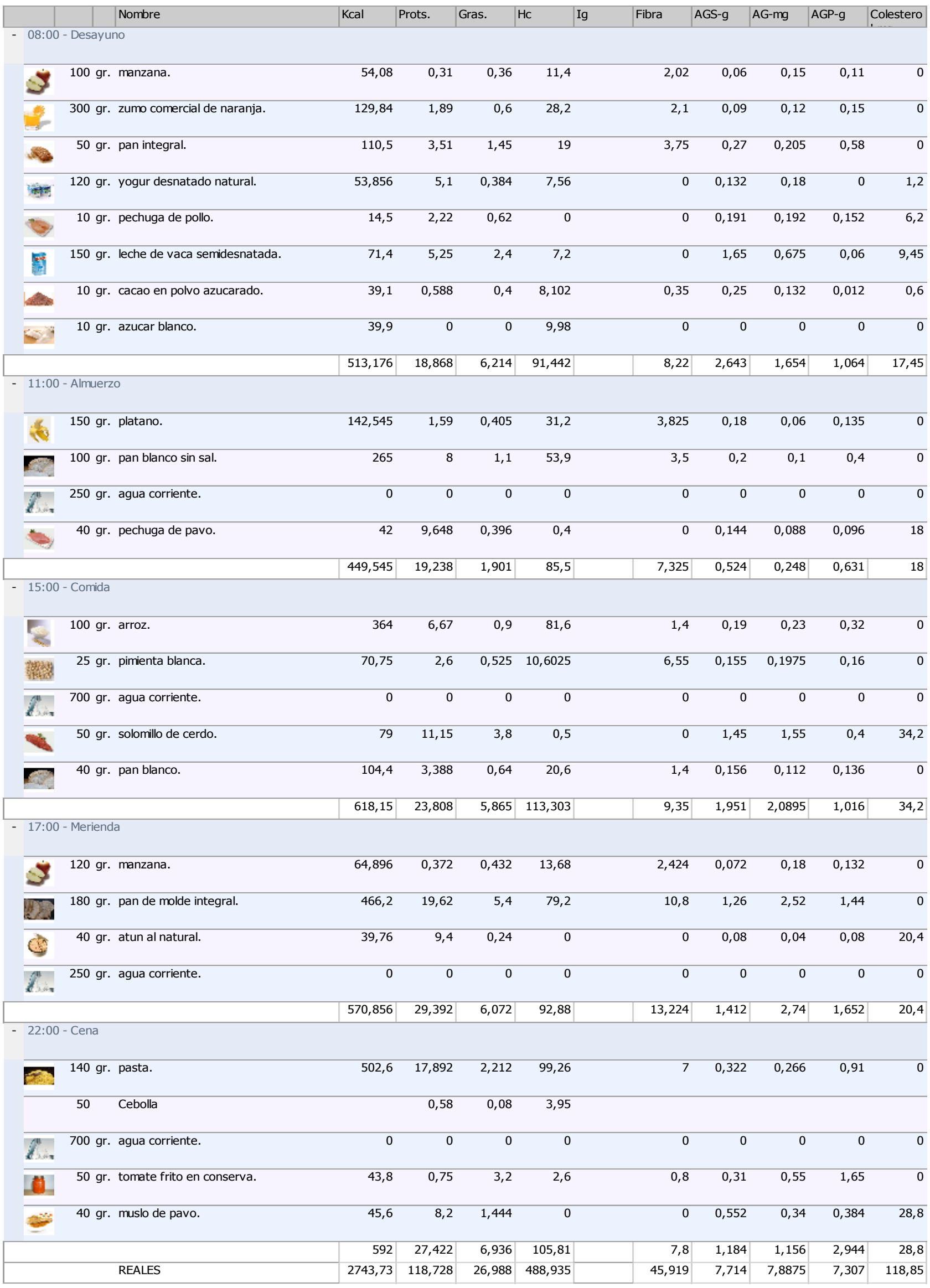




\section{Jueves}

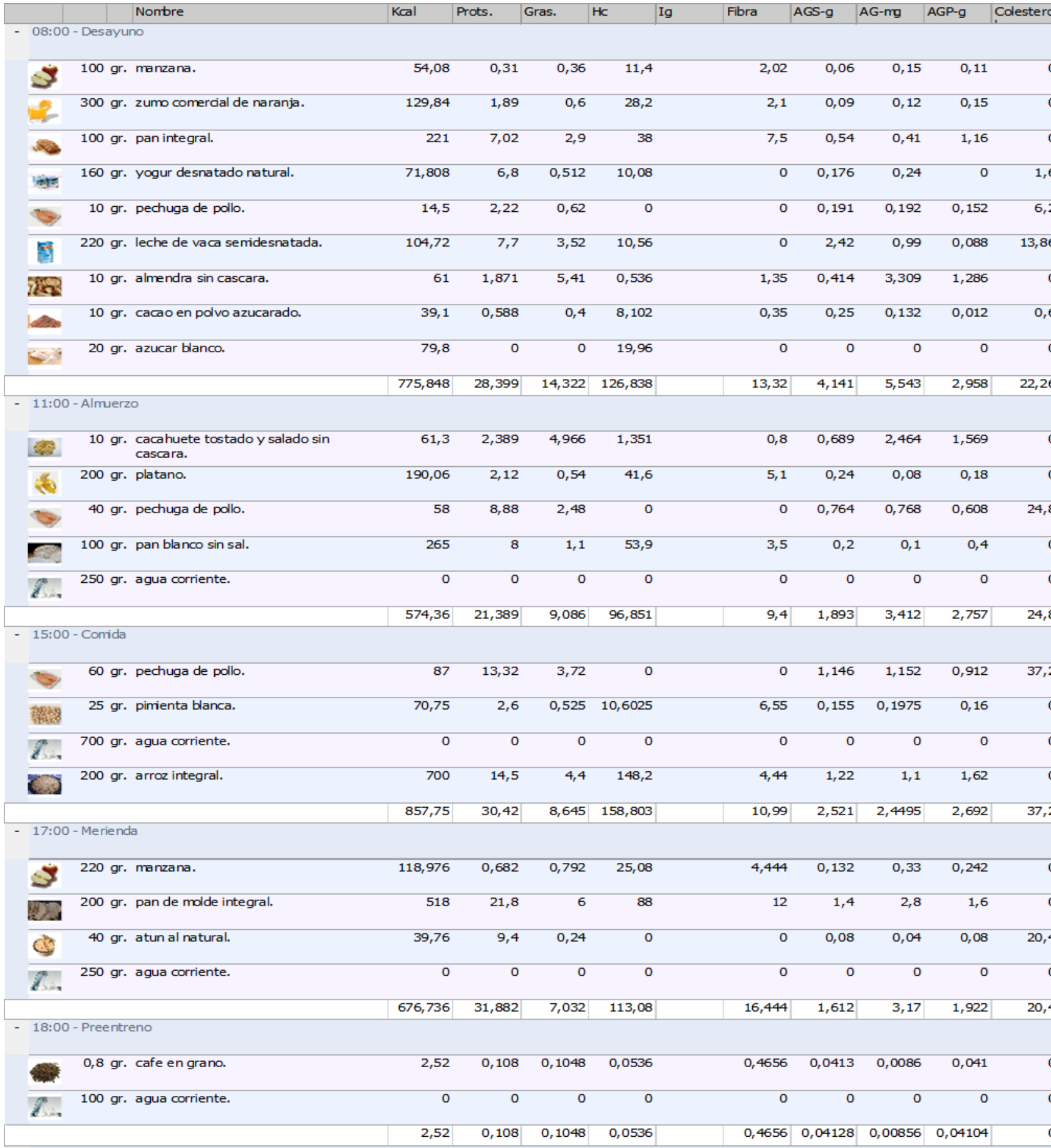

\section{9:00 - Entrenamiento}

\section{|t| 200 Powerade ION4} $34 \quad 7,8$

REALES FALTA O EXCESO

Número 3, 2014

\begin{tabular}{|r|r|r|r|r|r|r|r|r|} 
& 0,58 & 0,08 & 3,95 & & & & & \\
\hline 0 & 0 & 0 & 0 & & & & & \\
& & & & & & & & \\
\hline 785,4 & 25,935 & 8,82 & 146,79 & 7,098 & 1,89 & 3,15 & 0,84 & 63 \\
\hline 835,1 & 27,775 & 10,16 & 158,86 & 7,518 & 2,1 & 3,71 & 1,33 & 63 \\
\hline 3756,31 & 139,973 & 49,3498 & 662,285 & 58,1376 & 12,3083 & 18,2931 & 11,7 & 167,66 \\
\hline-7 & -1 & -14 & 4 & & & & & \\
\hline
\end{tabular}

E-motion. Revista de Educación, Motricidad e Investigación 
- Dieta periodo 3: Competición (Mayo).

Durante este periodo como he mencionado anteriormente, venimos de una bajada de peso considerable para competir con la máxima cantidad magra y el menor porcentaje de grasa corporal. Igualmente, el porcentaje de ingesta de la grasa debe ser alto para favorecer altos niveles de testosterona y que no exista una disminución repentina del nivel. Hay que destacar que esta dieta es solo para estos 4 días, ya que como se puede apreciar (teóricamente) más adelante la ingesta proteínica es muy baja, lo que a la larga sería realmente contraproducente (por ejemplo los niveles de creatinina). Hay que tener en cuenta los factores ambientales, porque aunque se presume una temperatura correcta, podemos encontrarnos con ciertas alergias y uso de fármacos debido a la época del año. Los 4 días elegidos serán:

- Lunes: Descanso.

- Martes: Descanso.

- Miércoles: Competición.

- Este día deberá tener comidas previas con un alto índice de kcal proveniente de los carbohidratos.

- Jueves: Descanso.

Necesidades calóricas días de competición: 1846 kcal/día *2,1 (intensidad - factor térmico ejercicio) $* 1,1($ factor termogénico $10 \%$ )= $4264.26 \mathrm{kcal}$

Necesidades calóricas días de descanso: 1846 kcal/día * 1,6 * 1,1 = 3248.96 kcal Reparto de macronutrientes:

70\% Hc; $20 \%$ Grasas; $10 \%$ Proteína

746 g Hc; 95 g Grasas; 106 g Proteína

568 g Hc; 72 g Grasas; 81 g Proteína 


\section{Lunes}

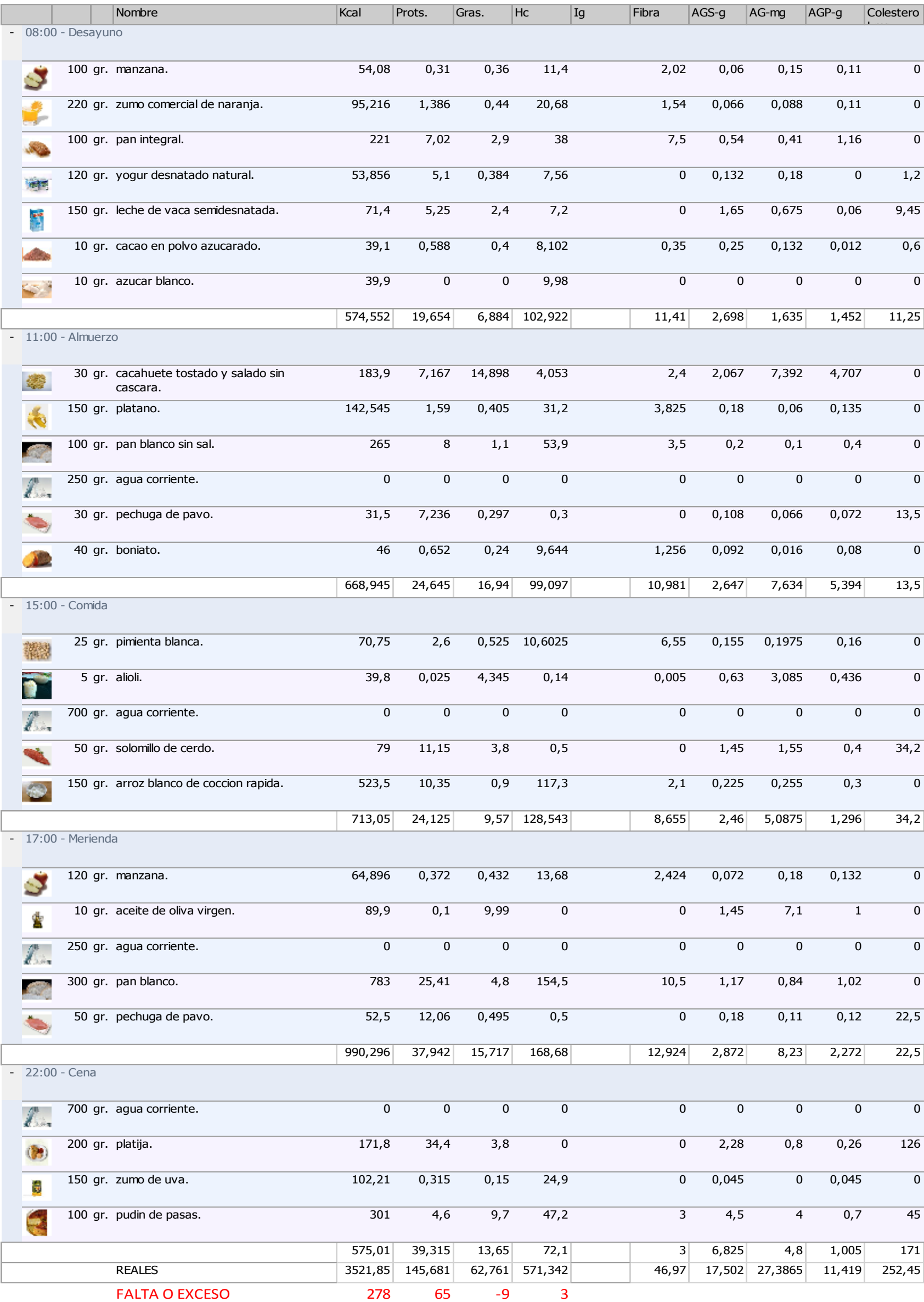




\section{Martes}

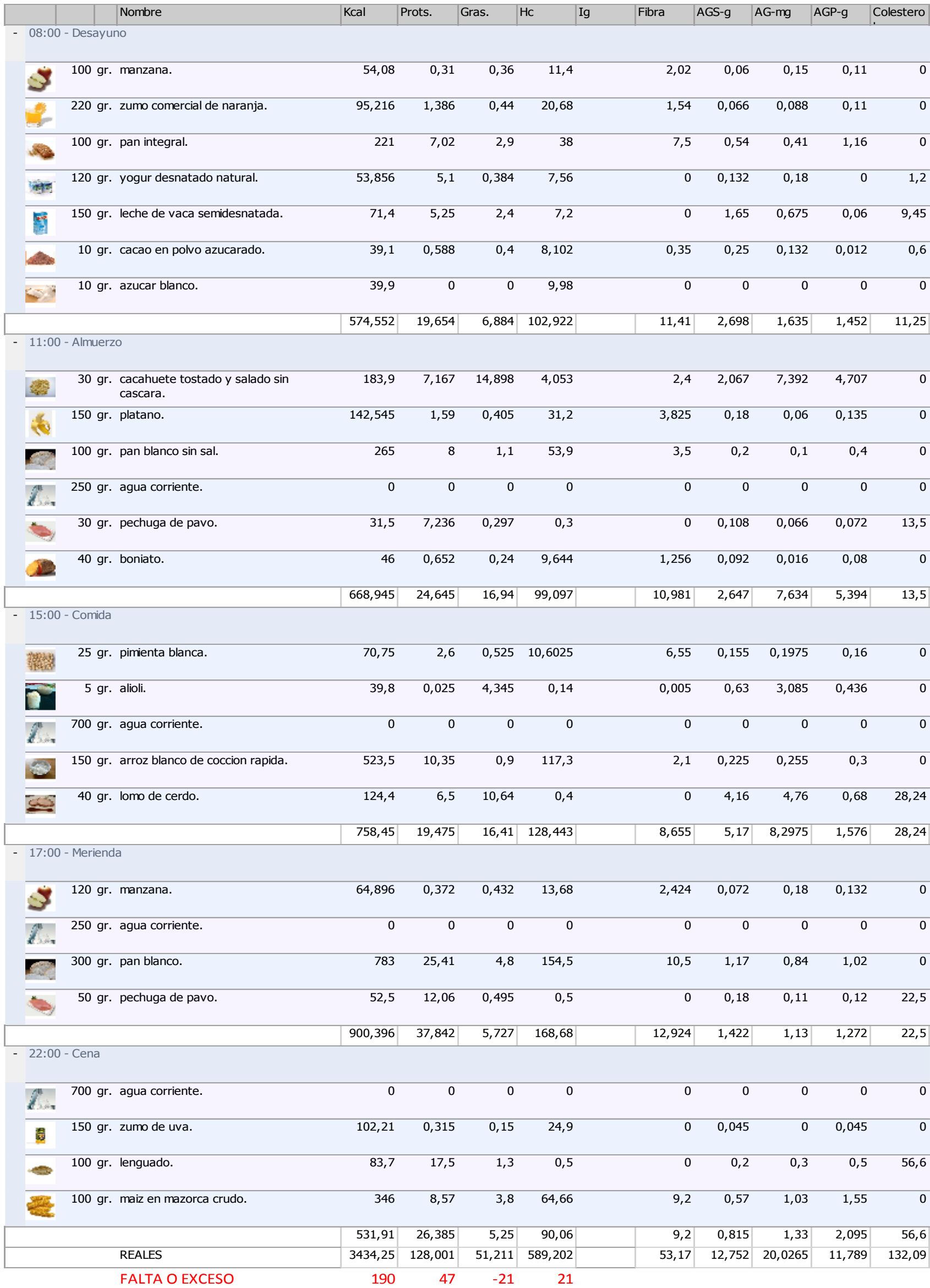




\section{Miércoles}

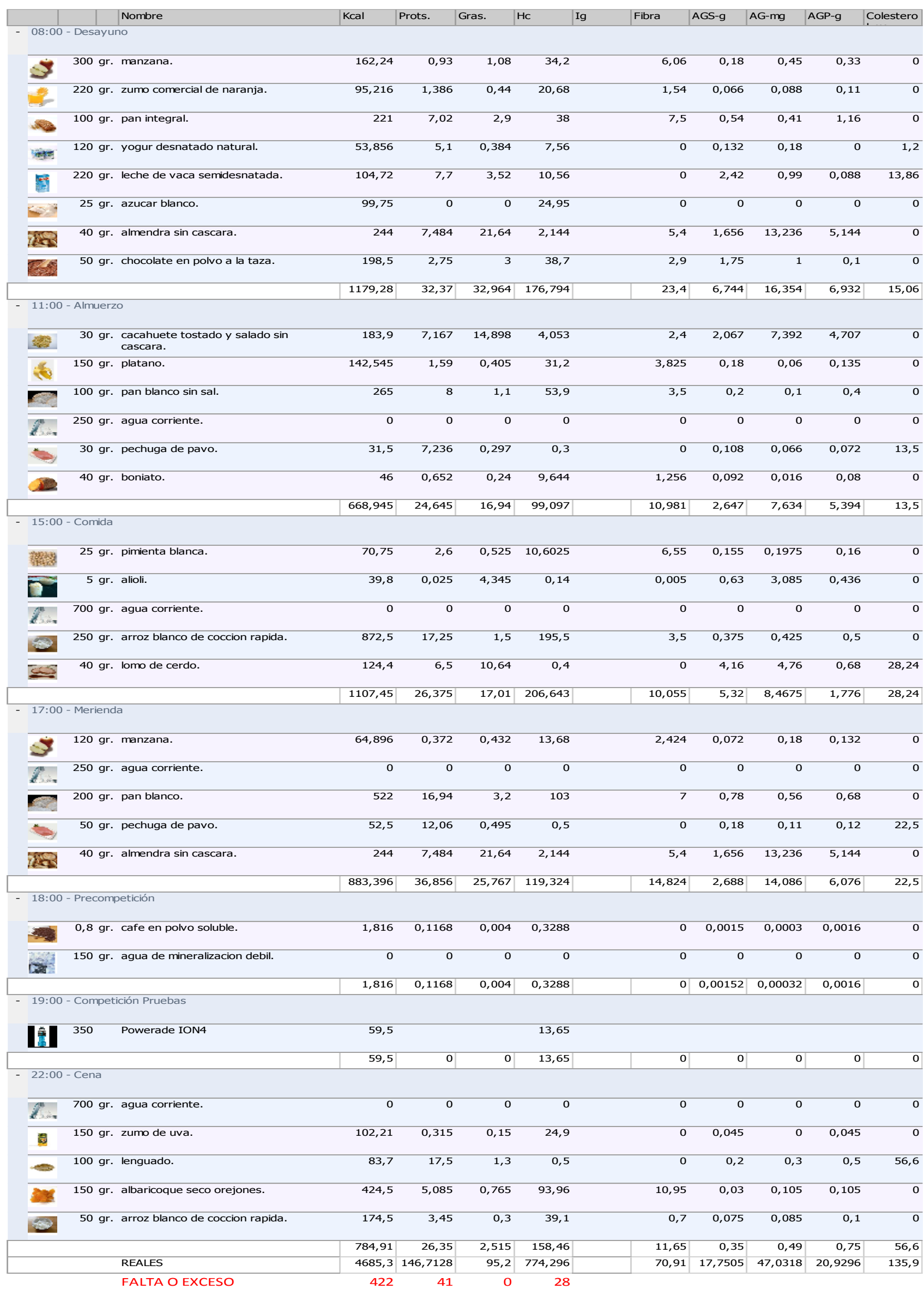




\section{Jueves}

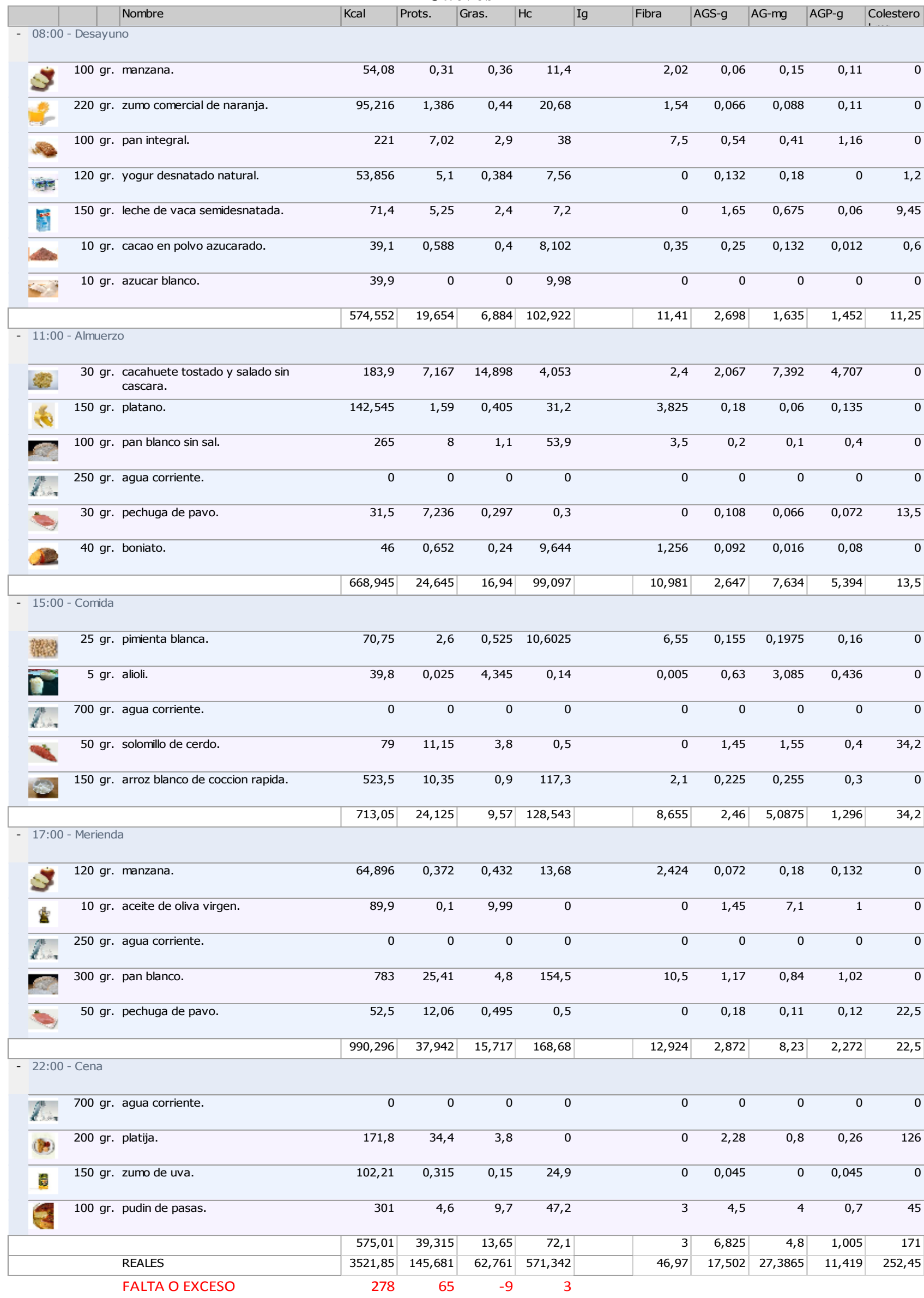


- Dieta periodo 4: Lesión (Junio).

Durante este periodo como he mencionado anteriormente, el sujeto tiene una lesión presumiblemente en la musculatura ejecutora de las flexiones de dedo y rotación del antebrazo que le impide mantener el rendimiento de competición, pero al solo tener una inmovilización parcial del antebrazo el entrenamiento disminuye la intensidad junto con la eliminación de algunos ejercicios pero mantiene su constancia, lo que ayudará a la recuperación. Igualmente, el porcentaje de ingesta de la grasa debe ser alto para favorecer altos niveles de testosterona pero teniendo especial cuidado respecto a las vitaminas, ya que algunas ayudarán a la recuperación y otras aumentarán la sensación de dolor (preferencia por hortalizas, frutas y pescados). Hay que tener en cuenta las condiciones ambientales ya que las temperaturas comienzan a elevarse (10\% más de la metabolismo basal). Los 4 días elegidos serán:

- Lunes: High interval power training (HIIT) en bicicleta.

- Martes: Descanso.

- Miércoles: HIIT en pista de atletismo.

- Jueves: Descanso.

Necesidades calóricas días de entreno: 1846 kcal/día * 1,1 (factor clima) * 1,8 (intensidad - factor térmico ejercicio) *1,1(factor termogénico $10 \%$ )= $4021 \mathrm{kcal}$ Necesidades calóricas días de descanso: $1846 \mathrm{kcal} /$ día $* 1,1 * 1,6 * 1,1=3574 \mathrm{kcal}$ Reparto de macronutrientes:

60\% Hc; $20 \%$ Grasas; $20 \%$ Proteína

603 g Hc; 89 g Grasas; 201 g Proteína

536 g Hc; 79 g Grasas; 178 g Proteína 


\section{Lunes}

\begin{tabular}{|c|c|c|c|c|c|c|c|c|c|c|}
\hline & Nombre & Kcal & Prots. & Gras. & $\mathrm{Hc}$ & Fibra & AGS-g & AG-mg & AGP-g & Colestero \\
\hline \multicolumn{11}{|c|}{ - 08:00 - Desayuno } \\
\hline & 200 gr. manzana. & 108,16 & 0,62 & 0,72 & 22,8 & 4,04 & 0,12 & 0,3 & 0,22 & 0 \\
\hline 5 & 220 gr. zumo comercial de naranja. & 95,216 & 1,386 & 0,44 & 20,68 & 1,54 & 0,066 & 0,088 & 0,11 & 0 \\
\hline & 120 gr. pan integral. & 265,2 & 8,424 & 3,48 & 45,6 & 9 & 0,648 & 0,492 & 1,392 & 0 \\
\hline ater & 160 gr. yogur desnatado natural. & 71,808 & 6,8 & 0,512 & 10,08 & 0 & 0,176 & 0,24 & 0 & 1,6 \\
\hline & 10 gr. cacao en polvo azucarado. & 39,1 & 0,588 & 0,4 & 8,102 & 0,35 & 0,25 & 0,132 & 0,012 & 0,6 \\
\hline & 20 gr. azucar blanco. & 79,8 & 0 & 0 & 19,96 & 0 & 0 & 0 & 0 & 0 \\
\hline & & 659,284 & 17,818 & 5,552 & 127,222 & 14,93 & 1,26 & 1,252 & 1,734 & 2,2 \\
\hline \multicolumn{11}{|c|}{ - 11:00 - Almuerzo } \\
\hline & 150 gr. platano. & 142,545 & 1,59 & 0,405 & 31,2 & 3,825 & 0,18 & 0,06 & 0,135 & 0 \\
\hline & 120 gr. pechuga de pollo. & 174 & 26,64 & 7,44 & 0 & 0 & 2,292 & 2,304 & 1,824 & 74,4 \\
\hline $\bar{c}$ & 250 gr. agua corriente. & 0 & 0 & 0 & 0 & 0 & 0 & 0 & 0 & 0 \\
\hline 57 & 50 gr. pan blanco tipo baguette. & 129 & 4,825 & 0,55 & 25,3 & 1,75 & 0,1 & 0,05 & 0,2 & 0 \\
\hline & & 445,545 & 33,055 & 8,395 & 56,5 & 5,575 & 2,572 & 2,414 & 2,159 & 74,4 \\
\hline \multicolumn{11}{|c|}{ - 15:00 - Comida } \\
\hline 5 & 200 gr. arroz. & 728 & 13,34 & 1,8 & 163,2 & 2,8 & 0,38 & 0,46 & 0,64 & 0 \\
\hline 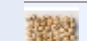 & 25 gr. pimienta blanca. & 70,75 & 2,6 & 0,525 & 10,6025 & 6,55 & 0,155 & 0,1975 & 0,16 & 0 \\
\hline 6 & 700 gr. agua corriente. & 0 & 0 & 0 & 0 & 0 & 0 & 0 & 0 & 0 \\
\hline$\overline{5}$ & 150 gr. pez espada. & 175,5 & 29,025 & 6,6 & 0 & 0 & 1,755 & 2,49 & 1,725 & 58,5 \\
\hline & 150 gr. tomate. & 33,255 & 1,32 & 0,315 & 5,25 & 2,1 & 0,06 & 0,045 & 0,15 & 0 \\
\hline 驲 & 100 gr. guisante congelado. & 74 & 6 & 0,4 & 9,51 & 4,2 & 0,15 & 0,13 & 0,05 & 0 \\
\hline & & 1081,51 & 52,285 & 9,64 & 188,563 & 15,65 & 2,5 & 3,3225 & 2,725 & 58,5 \\
\hline \multicolumn{11}{|c|}{ - 17:00 - Merienda } \\
\hline & 200 gr. manzana. & 108,16 & 0,62 & 0,72 & 22,8 & 4,04 & 0,12 & 0,3 & 0,22 & 0 \\
\hline$\sqrt{18}$ & 180 gr. pan de molde integral. & 466,2 & 19,62 & 5,4 & 79,2 & 10,8 & 1,26 & 2,52 & 1,44 & 0 \\
\hline ats & 120 gr. atun al natural. & 119,28 & 28,2 & 0,72 & 0 & 0 & 0,24 & 0,12 & 0,24 & 61,2 \\
\hline$\overline{7}$ & 250 gr. agua corriente. & 0 & 0 & 0 & 0 & 0 & 0 & 0 & 0 & 0 \\
\hline & & 693,64 & 48,44 & 6,84 & 102 & 14,84 & 1,62 & 2,94 & 1,9 & 61,2 \\
\hline
\end{tabular}

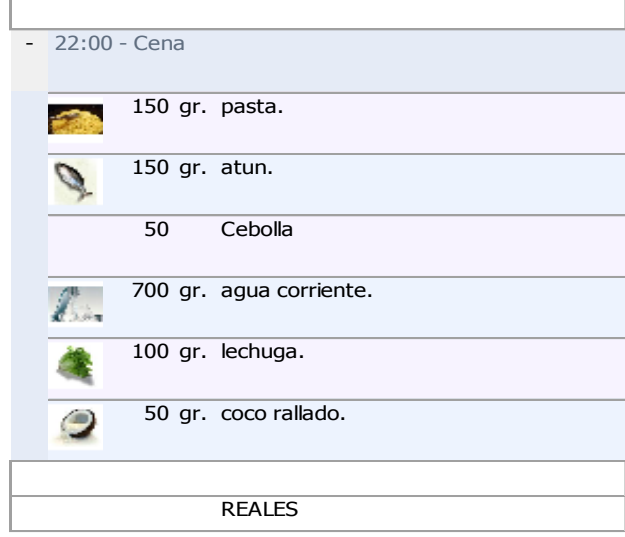

\section{FALTA O EXCESO}

Número 3, 2014

\begin{tabular}{|l|l|l|l|}
\hline 34 & 0 & 0 & 7,8 \\
\hline
\end{tabular}

$\begin{array}{lllllllll}538,5 & 19,17 & 2,37 & 106,35 & 7,5 & 0,345 & 0,285 & 0,975 & 0\end{array}$

$\begin{array}{lllllllll}339 & 32,25 & 23,25 & 0 & 0 & 6,225 & 5,145 & 6,63 & 72\end{array}$

$0 \quad 0$

$19,6 \quad 1,37 \quad 0,6 \quad 1,4$

$\begin{array}{lllll}1,5 & 0,12 & 0,01 & 0,37 & 0\end{array}$

$316,5 \quad 2,8 \quad 31 \quad 3,2$

\begin{tabular}{|r|r|r|r|}
\hline 1213,6 & 56,17 & 57,3 & 114,9 \\
\hline 4127,57 & 207,768 & 87,727 & $596,985 \mid$
\end{tabular}

\begin{tabular}{|l|l|r|r|}
\hline 4127,57 & 207,768 & 87,727 & 596,985 \\
\hline
\end{tabular}

\begin{tabular}{|r|r|r|r|r|}
\hline 15,85 & 33,39 & 7,19 & 8,725 & 72 \\
\hline
\end{tabular}

$\begin{array}{llll}111 & 7 & -1 & -6\end{array}$

E-motion. Revista de Educación, Motricidad e Investigación 


\section{Martes}

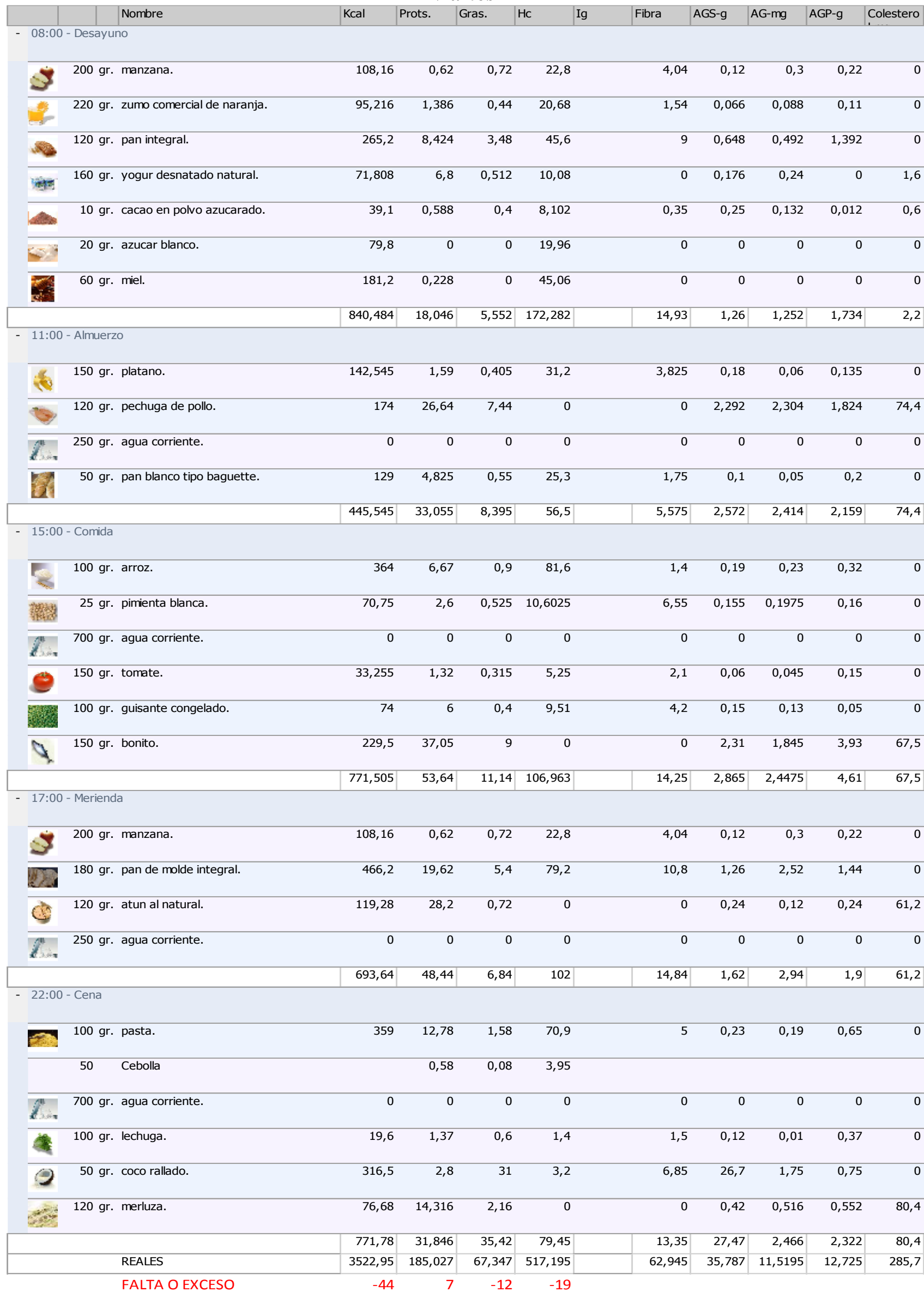

FALTA O EXCESO

Número 3, 2014
E-motion. Revista de Educación, Motricidad e Investigación 


\section{Miércoles}

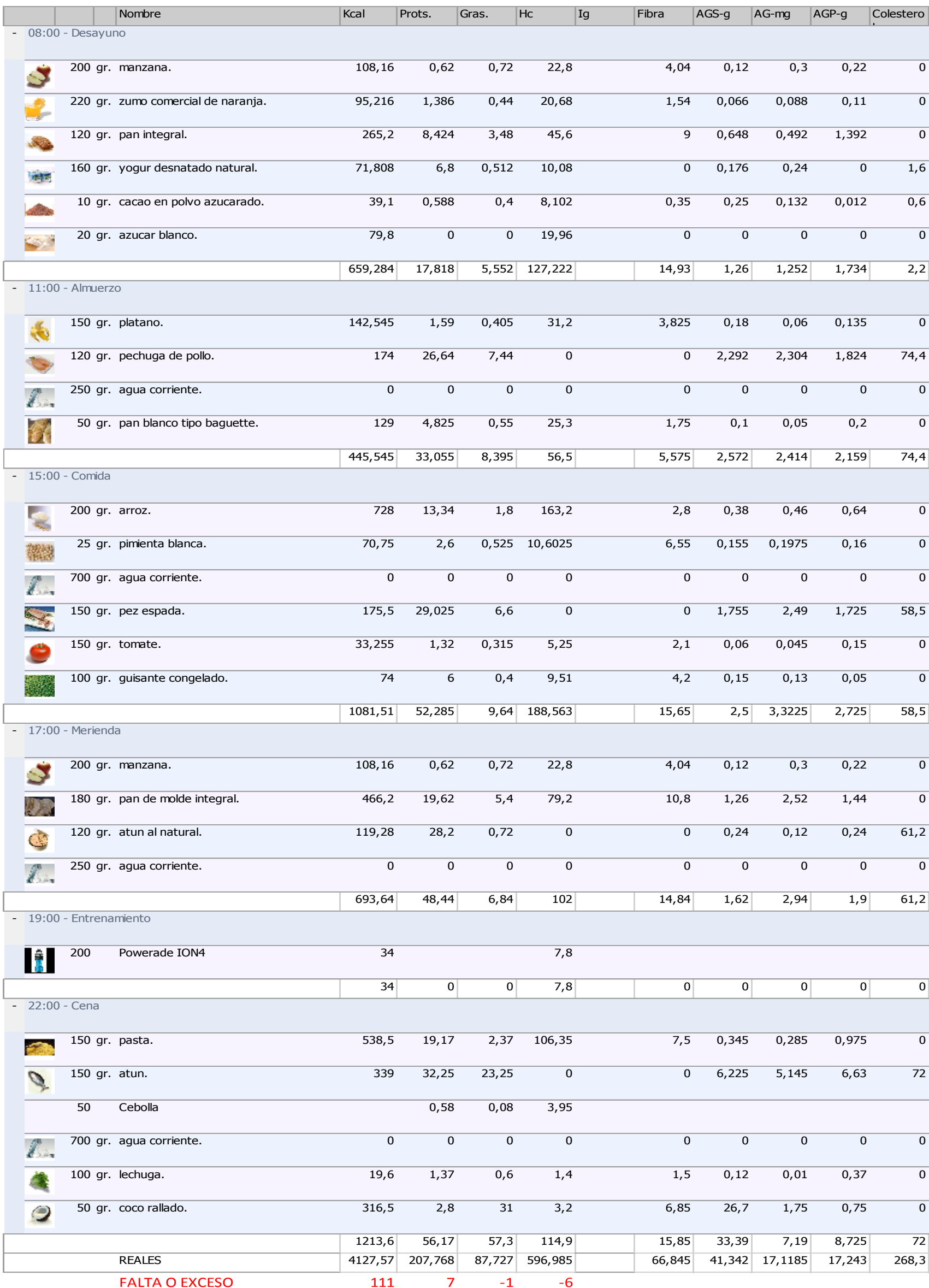




\section{Jueves}

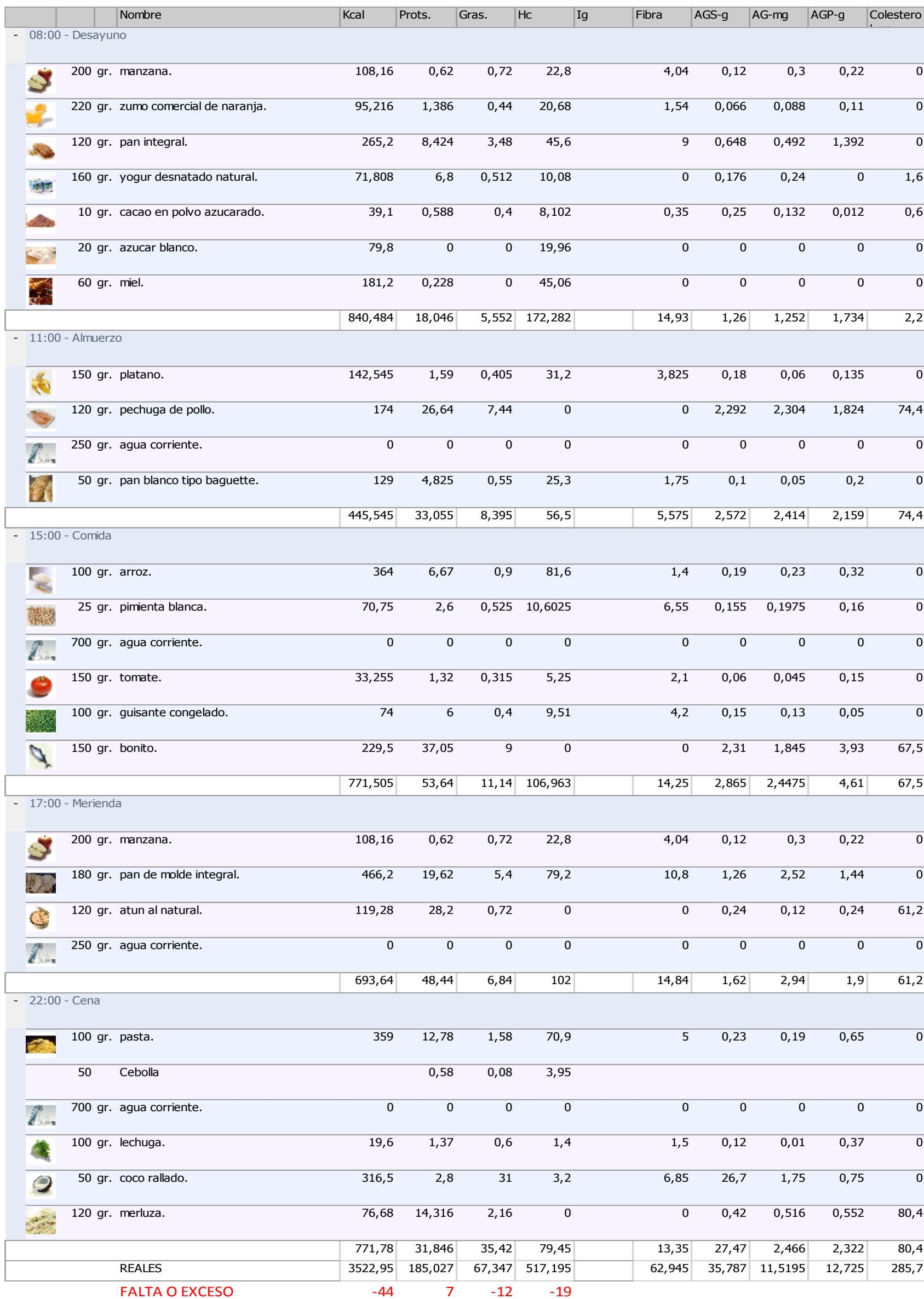




\section{BIBLIOGRAFÍA}

- American College of Sports Medicine (1996). Position Stand: Exercise and fluid replacement. Medicine \& Science in Sports \& Exercise. № 29, pp. 1-11.

- Artero, E.G., Castilllo, M.J., España-Romero, V., Gutiérrez, A., Jiménez-Pavón, D., Ortega, F.B. y Ruiz, J.R. (2009). Aspectos fisiológicos de la escalada deportiva. Revista Internacional de Medicina y Ciencias de la Actividad Física y el Deporte. Vol. 9, No35, pp. 264-298.

- Baechle, T.R., y Earle, R.W. (2008). Manual NSCA. Fundamentos del entrenamiento personal. Barcelona: Paidotribo.

- Barrios, V., Guerrero, J., Martínez-Sanz, J.M., y Urdampilleta, A. (2011). Lecturas: Educación Física y Deportes. Extraído el 7 de mayo de 2014 de: http://www.efdeportes.com/efd159/el-somatotipo-morfologia-en-losdeportistas.html

- Barrios, V., Guerrero, J., Otegui, A.U., y Sanz, J.M.M. (2011). El somatotipomorfología en los deportistas. ¿Cómo se calcula? ¿Cuáles son las referencias internacionales para comparar con nuestros deportistas? Lecturas: Educación física y deportes, (159), 4.

- Boetes, M., Bush, J.A., Incledon, T., Kraemer, W.J., y Volek, J.S. (1997) Testosterone and cortisol in relationship to dietary nutrients and resistance exercise. Journal of Applied Physiology. No 82, pp. 49-54.

- Castillo, M.J., Gutiérrez, A., Mesa, J.L., Mula, F.J., y Ruiz, J.R. (2003). Hidratación y rendimiento: pautas para una elusión efectiva de la deshidratación por ejercicio. Apunts: Educación Física y deportes (submitted 2001). № 70, pp. 26-33.

- Cooper, L.W., Feagin Jr, J.A., Glisson, R.R., Koukoubis, T.D., y Seaber, A.V. (1995). An electromyographic study of arm muscles during climbing. Journal: Knee Surgery Sports Traumatology Arthroscopy, 3(2), 121-124. Extraído el 24 de marzo de 2014 de: http://link.springer.com/article/10.1007/BF01552388

- Devor, S., Smith, M., Sommer, A., y Starkoff, B. (n.d.). Crossfit-based high intensity power training improves maximal aerobic fitness and body composition. Extraído el 17 de junio de 2014 de: http://library.crossfit.com/free/pdf/CFJ_Devor_CrossFit_Publication_1.pdf 
- FAO. (1985). Necesidades de energía y de proteínas. Ginebra, (Serie de Informes Técnicos. $\mathrm{N}^{\mathrm{o}} 724$.

- Fernández Rosa, L., y Herrero de Lucas, A (n.d.). La cineantropometría en los deportes de fuerza (halterofilia, judo y lucha). Extraído el 13 de marzo de 2014 de: http://cdeporte.rediris.es/revista/revista35/artescalada129.html

- Martínez de Haro, V. (2010). Aplicación del "cubo de la salud" en la readaptación de lesiones en fútbol profesional. En V. Martínez de Haro (Comp). IV Congreso internacional universitario de las ciencias de la salud y el deporte. (pp. 345-346). Madrid, España: Universidad autónoma de Madrid.

- Nieto Martínez, R.E. (s.f) Termogénesis y balance energético. Universidad centroccidental "Lisandro Alvarado".

- Rodríguez, A. (2014). Comparación de tasa metabólica en reposo medida por calorimetría indirecta versus ecuaciones predictivas, en mujeres adultas jóvenes y adultas mayores de peso corporal normal. Revista chilena de nutrición, 41(1), pp. 1722. 